\title{
Toward Predicting Prosocial Behavior: Music Preference and Empathy Differences Between Late Adolescents and Adults
}

\author{
SHANNON S. CLARK [1] \\ Australian Catholic University \\ S. GIAC GIACOMANTONIO \\ University of Queensland
}

\begin{abstract}
Empathy plays a role in social competence and intelligence, and can serve as a buffer against antisocial tendencies. Numerous studies highlight the relationship between empathy, prosocial behaviors, and the predictive utility of music preferences. This study examined participant differences in music preferences and empathy as a function of age, and whether preferred music genre predicted empathy (as a correlate to prosocial behavior). A new measure was devised to assess music preferences more accurately (i.e. with better face/construct validity) than existing measures. The Basic Empathy Scale measured empathy as a multidimensional construct. Younger participants exhibited greater empathy than older ones. Each music preference factor contributed uniquely to empathy variance in multiple regression models. Younger and older participants differed on music preferences (arguably associated with age-related sociocultural influences). Conclusions were drawn regarding the age differences in empathy and music preferences, the systematically greater influences of music preferences on cognitive compared to affective empathy, and the greater associations with empathy of specific music preferences. Limitations and implications for government policy and further research are considered.
\end{abstract}

Submitted 2014 May 13; accepted 2014 October 28.

KEYWORDS: music preferences, age comparisons, empathy, lyrical influence

\section{PROSOCIAL BEHAVIORS AMONG LATE ADOLESCENTS}

AFTER the recent floods in Brisbane, Australia (January 2011), disaster-relief and media organizations reported unusual, but welcome, volunteer assistance from early and late adolescents. The Australian and The Brisbane Times newspapers (January 15, 2011) both reported that this "helping behavior" was giving youths a greater sense of power to make a difference in their-and other-communities, than they had had previously. Similarly, The Herald Sun (January 18, 2011) and The Brisbane Times (January 21, 2011) highlighted the plight of several 20 year olds wanting to assist flood victims. This, they reported, was accomplished in several ways, most noticeably by organizing a benefit concert for other youths which attracted popular Australian artists The Veronicas. These media reports of an increased prosocial behavior among late adolescents are supported by statistics from the Volunteering Queensland Organisation, who reported the registration of close to 80,000 volunteers during the month of January. Of these volunteers 2,400 were under the age of $18(3 \%)$, and 24,800 were aged between 18 and $24(31 \%)$.

This rise in prosocial behaviors exhibited among late adolescents is echoed by the Australian Bureau of Statistics (ABS). In the ABS' results from the 2008 "Voluntary Work Survey," volunteering trends for ages 18-24 had increased from $17 \%$ in 1995 to $34 \%$ in 2008 (of 5.2 million volunteers Australia wide). $26 \%$ of this age bracket (the largest percentage) indicated their motivating factor as "wanting to help others and give back to the community," and $22 \%$ (the second largest percentage) indicated their motivating factor as "wanting to gain life experience." Although the above-mentioned reports have focused on a particular geographic region and data on volunteering may not have been collected systematically over 
a long period of time, the findings of these reports nevertheless strongly suggest that late adolescents are exhibiting increases in collective prosocial behavior and it is deliberate.

\title{
WHAT ARE PROSOCIAL BEHAVIORS, AND HOW DO THEY DEVELOP?
}

\author{
"A prince must learn how not to be good." \\ -Machiavelli (1532/2008)
}

Before the 1970s, "altruism" was the term most commonly used for the behaviors under discussion. Then, in a 1972 issue of the Journal of Social Issues, Wispé coined the term "prosocial" as an antonym to the well-received term "antisocial." It has since been argued that if violence and aggression were seen as antisocial, then their opposites could be seen as prosocial (Clark \& Giacomantonio, 2013; Hay, 1994). A consensus exists within contemporary developmental psychology that prosocial behaviors include any actions that promote harmonious relations with, and/or benefit others. Examples include helping, sharing, cooperation (Nantel-Vivier et al., 2009), sympathy, and comforting (Scourfield, John, Martin, \& McGuffin, 2004). Prosocial behaviors that facilitate positive social relations are thought to decline during early adolescence (Scourfield et al., 2004), when children learn how to maintain a balance between their selfinterests and their prosocial impulses (Hay, 1994), and then increase again with the maturation of empathic concern during late adolescence (Clark \& Giacomantonio, 2013; Eisenberg \& Fabes, 1998; Nantel-Vivier et al., 2009). Therefore, it appears that children, like Machiavelli's Prince, learn how to identify the specific circumstances in which they need not be prosocial (Hay, 1994), restricting prosocial behaviors to their affiliate groups (i.e. their in-groups), which include their friends and family during this period of adolescence (Nantel-Vivier et al., 2009).

\section{What is Empathy?}

In current theories on empathic understanding, some researchers have defined empathy as a unique capacity in humans to feel the experiences of others as their own (e.g. Clark, 1980; Cohen \& Strayer, 1996; Crenshaw \& Hardy, 2007; Hoffman, 2001; LeSure-Lester, 2000). Empathy is a higher-order human attribute, believed to be especially important in the moral development of adolescents (Kassin, Fein, \& Markus, 2006; Sigelman \& Rider, 2009).

Modern developmental theorists (e.g. Clark \& Giacomantonio, 2013; Cohen \& Strayer, 1996; Crenshaw \& Hardy, 2007; Epley, Savitsky, \& Golovich, 2002; Hoffman, 2001) postulate that empathy should be considered a multidimensional construct consisting of two broad components: (a) a cognitive component: perspective taking, and (b) an emotional component: vicarious experiencing. The major component of cognitive empathy (i.e. perspective taking) entails using the power of imagination to try to see the world through someone else's eyes; the major component of affective empathy (i.e. vicarious experiencing) entails the ability to vicariously experience someone else's emotional state and/or feelings (Clark \& Giacomantonio, 2013; Kassin et al., 2006; Sigelman \& Rider, 2009). It is also suggested that a well-rounded grasp of empathic concern involves the joint operation of both the affective and cognitive components (Caravita, Di Blasio, \& Salmivalli, 2009; Clark \& Giacomantonio, 2013; Cohen \& Strayer, 1996; Hoffman, 2001).

\section{Why Investigate Empathy?}

In current theories on the development of prosocial and antisocial behaviors, empathy has been recognized as one of the most important individual difference characteristics (de Kemp et al., 2007). Empathy is suggested to play a role in the acquisition of social competence during adolescence (Clark \& Giacomantonio, 2013). Moreover, empathy has been positively related to social intelligence and is suggested to serve as a buffer for all forms of aggressive and antisocial tendencies in adolescence (Albiero, Matricardi, Speltri, \& Toso, 2009; Clark \& Giacomantonio, 2013). Previous research has linked deficiencies in empathy with increased levels of aggressive and antisocial behaviors (for a review see Clark \& Giacomantonio, 2013; Jolliffe \& Farrington, 2006a), and it is a frequently documented belief that empathy facilitates adaptive and prosocial behavior and reduces aggression and antisocial behaviors (LeSure-Lester, 2000). 
A lack of empathy, conversely, implies a predisposition toward prejudice (Albiero et al., 2009), and can be the basis of social tensions, and antisocial affect and behavior (Crenshaw \& Hardy, 2007). It has been suggested that children and adolescents displaying little empathy and concern for others care less about winning approval from, and are less influenced by praise from, authorities and peers (de Kemp et al., 2007). As a result, this is thought to potentially encourage antiauthoritarianism through the development of antisocial affect and subsequent antisocial behavior (Clark \& Giacomantonio, 2013; de Kemp et al., 2007).

\section{Previous Research in Empathy and Prosocial/Antisocial Behaviors}

Two decades ago, no systematic review concerning the relationship between empathy and aggression, and empathy and other antisocial behaviors had been conducted. At that time, Miller and Eisenberg (1988) conducted a meta-analysis of the existing literature. The primary objectives of their review were: to examine whether individuals who are aggressive in their interactions with others also tended to be lower in empathic responsiveness; to examine whether individuals who exhibit antisocial behavior differ from other people in empathic responsiveness. Fourteen relevant studies were found at the time, which revealed relatively clear evidence of a modest negative relation between empathy and aggressive and antisocial behavior, in both males and females.

Over the past decade, there have been a limited number of studies that provide support for the previously mentioned "empathy-prosocial/antisocial behavior" theories of Albiero et al. (2009), Crenshaw and Hardy (2007), and de Kemp et al. (2007). For a complete review of these studies, see Clark and Giacomantonio (2013) who highlight the systematic negative associations between empathy and antisocial behavior, and positive associations between empathy and prosocial behaviors.

Research on the effects of exposure to prosocial media on empathy, such as video games, has been relatively sparse. A 2010 study (Greitemeyer, Osswald, \& Brauer, 2010), using a non-clinical population, revealed that playing prosocial video games significantly increased empathic concern $(t[56]=2.24, p<.05)$ for both males and females; although females overall reported more empathy than males $(B=.36, t[49]=$ $2.82, p<.05)$. The researchers claimed that their results showed a systematic negative association between empathy and antisocial behavior, and it was suggested that these effects may not be limited to video game exposure but may also extend to other media sources such as music. For instance, it appears that listening to songs with prosocial lyrics can increase prosocial thoughts, affect, and behavior (Greitemeyer, 2009a, 2009b). It was suggested in these studies, that investigations into the prosocial behavior-empathy association may highlight the importance of examining ways to increase empathy in order to instigate helping behavior (Greitemeyer et al., 2010).

\section{How Best to Measure Empathy?}

Considering that empathizing involves two broad elements (i.e. cognitive and affective), it seems prudent for any studies looking into empathy to approach the construct in a multidimensional manner (Davis, 1980). Contemporary empathy researchers claim that the Interpersonal Reactivity Index (IRI; Davis, 1980, 1983) has certain shortcomings (e.g. Jolliffe \& Farrington, 2006a). Building on Davis's (1983) work, Jolliffe and Farrington (2006b) incorporated Davis's dimensions and produced an empathy measure that addressed the IRI's deficiencies. Consequently, the Basic Empathy Scale (BES) was devised. The similarities between the BES and the IRI is reflected in the relatively high inter-correlations for both the cognitive and affective empathy components $(r=.36 \& r=.52$ males, $\& r=.38 \& r=.35$ females respectively, significant at $p<.05)$ and total empathy scores $(r=.53$ males, $r=.43$ females $p<.05)$ (Jolliffe \& Farrington, 2006b).

\section{MUSIC PREFERENCES}

\section{Why Investigate Music Preferences?}

Music is the most omnipresent phenomenon of human society (for a review of studies highlighting this, see Clark \& Giacomantonio, 2013). Many people seek out music for their own listening purposes, and, taken together with background music, this can add up to more than a quarter of a Western individual's day; especially adolescents (Rentfrow, Goldberg, \& Levitin, 2011; Huron, 2001; Levitin, 2006; McCormick, 
2009; Roberts, Henriksen, \& Foehr, 2004). The importance of music is also reflected in the billions of dollars spent each year on: (a) music's consumption, and (b) music's construction by industry (Geter \& Streisand, 1995). With the advent of many new online options, music has never been easier to access in any setting.

Given that music is known to play an important role in such psychological functions as emotional regulation and coping, and also has the potential to evoke powerful emotional responses from its listeners (Juslin \& Laukka, 2003; Juslin \& Sloboda, 2001), it is not surprising that music-listening is shown to have implications for psychosocial adaptation, particularly for resolving developmental tasks. Examples include identity formation, social adaptation with peers, and emotion regulation (Miranda \& Claes, 2008).

Although the music an individual chooses to consume (i.e. their music preference) has received relatively little attention in mainstream social, and developmental psychology, recent investigations have begun to examine individual differences in music preferences (for a review, see Rentfrow, McDonald, \& Oldmeadow, 2009). However, additional research that validates music preferences as a measure of developmental accomplishments and issues is needed (Rentfrow, Goldberg, \& Levitin, 2011). Exposing individuals to a specific variety or genre of music may promote greater self-exploration, validation, and normalization of their psychosocial, psychological, and developmental issues, thus enhancing their social development (Schwartz \& Fouts, 2003).

As recent research shows (see Rentfrow \& Gosling, 2003; Colley, 2008; Schäfer \& Sedlmeier, 2009; Dunn, 2012; George et al., 2007; Leung \& Kier, 2008), there have been numerous ways that types and genres of music have been defined, classified, and categorized. However, despite the subtle differences within the extant literature there does appear to be a considerable degree of convergence, with several robust music-preference factors emerging that are very similar (Rentfrow \& Gosling, 2003). As a result, Rentfrow and Gosling devised the Short Test Of Music Preferences (STOMP) in order to measure an individual's consumption habits (or preferred music genre), and revealed four overarching music preference factors. They labeled these factors: Factor 1, "reflective \& complex" (comprising classical, jazz, folk, and blues genres); Factor 2, "intense \& rebellious" (comprising rock, alternative, and heavy metal genres); Factor 3, "upbeat \& conventional" (comprising country, pop, soundtracks, and religious genres); and Factor 4, "energetic \& rhythmic" (comprising rap, soul, dance, and electronica genres).

Over the past decade music preferences have been shown to influence and predict adolescents' behavioral patterns and attitudes (Leung \& Kier, 2008; Mulder et al., 2010; Rentfrow \& Gosling, 2006; Selfhout, Branje, ter Bogt, \& Meeus, 2009), identity formation (Sprankle \& End, 2009; Wester, Crown, Quatman, \& Heesacker, 1997), and psychopathology (Martin, Clarke, \& Pearce, 1993; Miranda \& Claes, 2008; Schwartz \& Fouts, 2003). These influences, which vary with age and gender, range from friendship formation, sexual acts, and substance use to civic activism, depression, and suicide (for a full review see Clark \& Giacomantonio, 2013). Here, we intended to seek evidence that the influence of music preferences extended to facets of emotional intelligence; namely empathy.

\begin{abstract}
Aims
As such, the aims of this exploratory study were to examine: (a) differences between late adolescents and adults for music preferences and empathy; (b) in what capacity (i.e. strength and direction) music preferences might be related to empathy; (c) whether age and/or gender are mitigating factors in those relationships; and (d) how well preferred music genre is able to predict cognitive, affective, and overall levels of empathy as an indicator of prosocial behaviors.
\end{abstract}

\title{
Hypotheses
}

It was hypothesized that late adolescents would have greater empathy scores than adults, due to their reported "empathy-maturation" period (see Eisenberg \& Fabes, 1998; Nantel-Viver et al., 2009). It was also hypothesized that these younger participants would have greater preferences, as scored on the researchers' own Music Preference Factor Scale (MPFS), for rebellious and energetic music than older participants, and that older participants would have greater preferences for reflexive and conventional music than would younger participants. In addition, it was hypothesized that the four music preference factors would have unique associations with empathic concern. Specifically, it was hypothesized that preferences for MPFS factors 1 (reflexive \& complex) and 2 (intense \& rebellious) would have a stronger 
association with empathy than MPFS factors 3 (upbeat \& conventional) and 4 (energetic \& rhythmic). Furthermore, it was hypothesized that age would influence the variance in those associations.

\section{METHOD}

\section{Participants and Sampling}

Participants were recruited in two ways: (1) via the social media site Facebook, and (2) via student email lists across two Australian Catholic University campuses (Brisbane and Melbourne). This created two kinds of samples: a snowball sample, and a convenience sample. For the latter group, participants were encouraged to participate by the offer of credit towards final semester grades.

The age cut-off for adulthood typically differs across studies (e.g. $c f$. Sigelman \& Rider, 2009, p. 4), and cultures (i.e. voting and drinking ages), so we coded participants to retain this age variation. In the present study, late adolescents were defined as participants aged 18-24, whilst participants aged $\geq 25$ were adults. The entire sample consisted of 322 participants (age range 18-56 years), Females $=237(73.6 \%)$ and Males $=85(26.4 \%)$, whose combined mean age was $M=27.38, S D=10.23$. We had a non-exclusive target sample of late adolescents and adults, both male and female, irrespective of cultural diversity. A power analysis suggested a target sample size of 146, based on a computation of the following figures: Total $N 50+8 m$, where $N 50$ for use of regression analysis, and where $m=$ number of independent variables (i.e. $50+[8 * 12]=146$, Tabachnick \& Fidell, 2007). The percentage of participants classified as "younger" (late adolescents) was $57.7 \%\left(n=173, M_{\text {age }}=20.11, S D=1.90\right)$, whilst the percentage of participants classified as "older" (adults) was $46.3 \%\left(n=149, M_{\text {age }}=35.83, S D=9.45\right)$.

\section{Materials}

Preferred music genre was measured using two separate assessments: the researchers' own MPFS, and the STOMP. The MPFS is a 32-item questionnaire capturing preferences for the 32 music genres set out in the Leung and Kier (2008) study. The MPFS asks participants to rate how often they listen to a specific genre. An example of an item would be "I like to listen to Classical music," and the item is endorsed along a 5point Likert scale where $1=$ "Not at all" and $5=$ "All the time." Scoring for the four music preference dimensions is as follows: Sub-Factor 1 comprises items 3, 4, 5, 12, and 18; Sub-Factor 2 comprises items 9, 11, 16, 17, 19, and 31; Sub-Factor 3 comprises items 15, 23, 25, 26, and 30; Sub-Factor 4 comprises items 6, 19, 22, and 32; Sub-Factor 5 comprises items 10, and 20; Sub-Factor 6 comprises items 1, 7, and 21; Sub-Factor 7 comprises items 14, 17, and 28; and Sub-Factor 8 comprises items 2, 8, and 13. The eight sub-factors load onto four broad-level factors. Factor 1 (MPF-1; reflexive and complex) comprises subfactors 1 and 4; Factor 2 (MPF-2; intense and rebellious) comprises sub-factors 6 and 7; Factor 3 (MPF-3; upbeat and conventional) comprises sub-factors 5 and 8; and Factor 4 (MPF-4; energetic and rhythmic) comprises sub-factors 2 and 3 (i.e. their respective counterparts from Rentfrow and Gosling [2003]), in accordance with the manual pairwise combination analysis. Scores are then interpolated into percentages of the total factor worth, and the factor with the highest recorded percentage among the four factors serves also as the general music preference factor.

The STOMP is a 14-item scale, assessing preferences in music genres. As described above, it assesses four broad music-preference dimensions: reflective and complex, intense and rebellious, upbeat and conventional, and energetic and rhythmic. The factor that scores the highest percentage of the total factor's worth represents the participant's music preference (for a full account, see Rentfrow \& Gosling, 2003). This measure was modified for use with online services.

Empathic concern was measured using the BES, a 20-item questionnaire that consists of 9 items addressing cognitive empathy, and 11 items addressing affective empathy (Jolliffe \& Farrington, 2006b).

\section{Procedure}

Having been invited to participate via email or Facebook invitation, participants were shown a hyperlink to an online survey, where they were presented with an information letter and consent option. At their convenience, participants provided responses to 66 items (not including demographic items) that comprised three separate measures: the MFPS, the STOMP, and the BES. Participation took no longer than 20 minutes 
if completed in one sitting. This study was approved by the National Human Research Ethics Committee (NHREC).

\section{Design}

"Preferred music genre" served as the independent variable, and was operationalized as participants' responses to the music preference questionnaire(s). "Empathy" served as the dependent variable, with two sub-specifications: (a) the cognitive component, considered "perspective-taking" (i.e. using the power of imagination to try to see the world through someone else's eyes); and (b) the affective component, considered "vicarious experiencing" (i.e. experiencing vicariously someone else's emotional state or feelings), both of which were measured by the BES Short Form. The research design was a within-subjects correlational study, assessing the relationship(s) between an individual's music preferences and their empathy levels.

\section{RESULTS}

\section{Music Preference and Empathy Scores}

At the beginning of this paper, it was mentioned that the MPFS would be used as the measure to capture an individual's preferred music genre. After reviewing the data between raw and percentage scores, and across measures (i.e. frequencies, descriptives, and correlations, presented in Figures 1 through 6, and Table 1), the researchers concluded that the MPFS raw scores captured music preferences across the samples with strong face- and criterion-related validity (see Kaplan \& Saccuzzo, 2005 for validity explanations). As such, MPFS raw scores were used for all subsequent analyses.

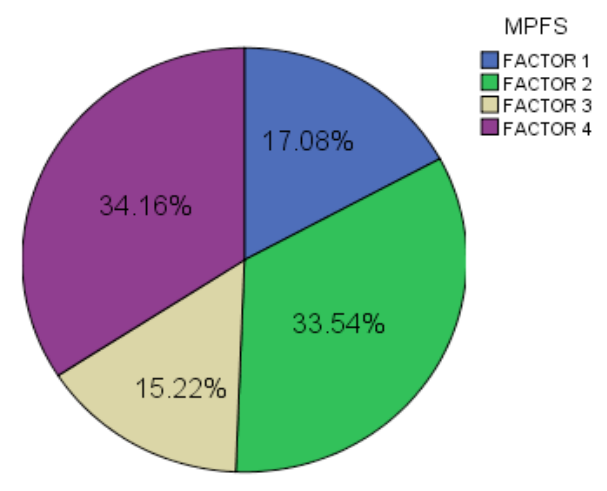

Figure 1. Music Factor Percentages: MPFS (entire sample)

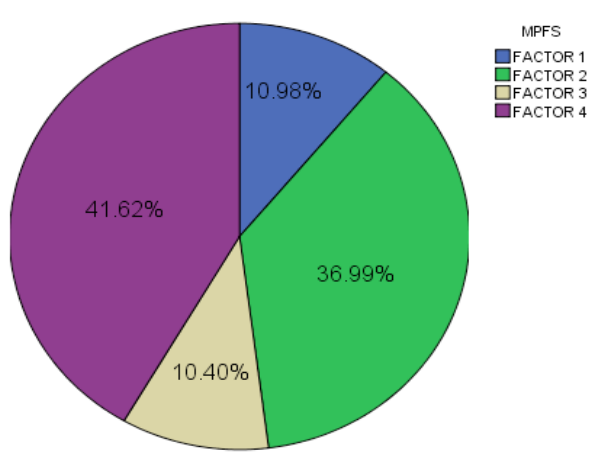

Figure 3. Music Factor Percentages

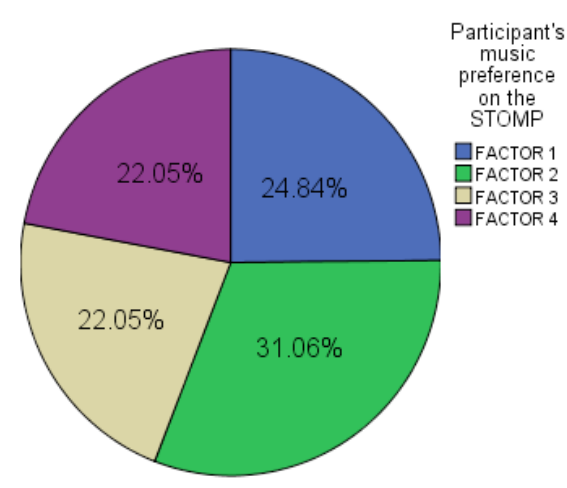

Figure 2. Music Factor Percentages: STOMP (entire sample)

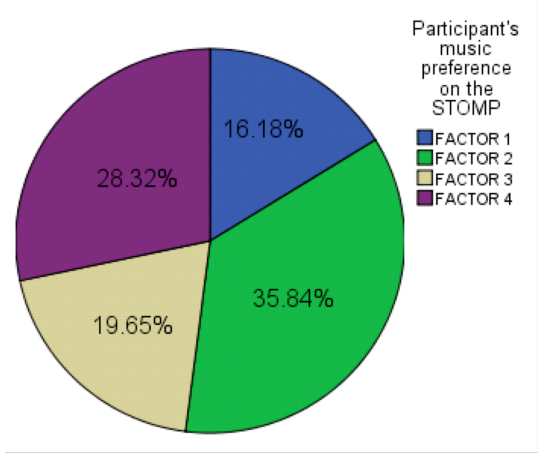

Figure 4. Music Factor Percentages 
MPFS (younger sample)

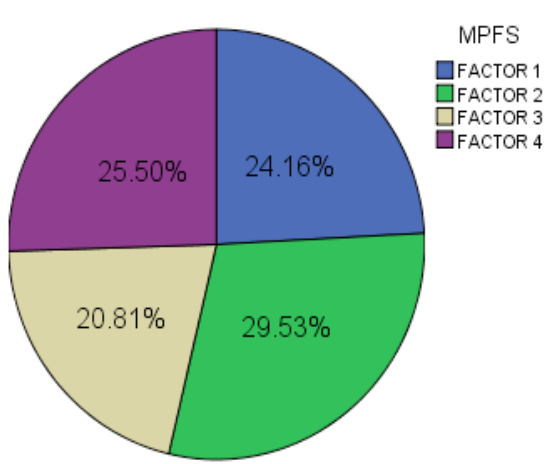

Figure 5. Music Factor Percentages MPFS (older sample)
STOMP (younger sample)

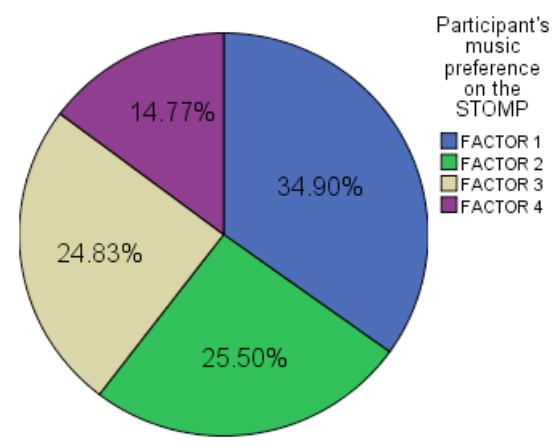

Figure 6. Music Factor Percentages STOMP (older sample)

Table 1. Music Preference Questionnaire Intercorrelations

\begin{tabular}{|c|c|c|c|c|}
\hline & MPF-1 & MPF-2 & MPF-3 & MPF-4 \\
\hline \multicolumn{5}{|l|}{ Entire Sample } \\
\hline STOMP Factor 1 & $.678 * *$ & & & \\
\hline STOMP Factor 2 & & $.727 * *$ & & \\
\hline STOMP Factor 3 & & & $.561 * *$ & \\
\hline STOMP Factor 4 & & & & $.730 * *$ \\
\hline \multicolumn{5}{|l|}{ Younger Sample } \\
\hline STOMP Factor 1 & $.616^{* *}$ & & & \\
\hline STOMP Factor 2 & & $.743 * *$ & & \\
\hline STOMP Factor 3 & & & $.493 * *$ & \\
\hline STOMP Factor 4 & & & & $.750 * *$ \\
\hline \multicolumn{5}{|l|}{ Older Sample } \\
\hline STOMP Factor 1 & $.739 * *$ & & & \\
\hline STOMP Factor 2 & & $.716^{* *}$ & & \\
\hline STOMP Factor 3 & & & $.621^{* *}$ & \\
\hline STOMP Factor 4 & & & & $.733 * *$ \\
\hline
\end{tabular}

$* * p<.01$

Means and standard deviations were calculated for each of the variables of interest. These were obtained for the sample as a whole and for the sample when split by age, and are presented in Table 2 . The preferred music factor for this sample as a whole was MPF-4 (energetic \& rhythmic), with $34.2 \%$ of participants choosing this as their preference (see Figure 1). However, when splitting the sample by age these figures are reflected differently. Whereas the younger participants still preferred MPF-4, and this percentage was greater $(41.6 \%$, see Figure 3), the older participants had a stronger preference for MPF-3 (29.5\%, see Figure 5). 
Table 2. MPFS and BES Means and Standard Deviations

\begin{tabular}{|c|c|c|c|c|c|c|}
\hline & \multicolumn{3}{|c|}{ Mean } & \multicolumn{3}{|c|}{$S D$} \\
\hline & $\mathrm{S}$ & $\mathrm{Y}$ & $\mathrm{O}$ & $\mathrm{S}$ & $\mathrm{Y}$ & $\mathrm{O}$ \\
\hline Age & 27.38 & 20.11 & 35.83 & 10.23 & 1.90 & 9.45 \\
\hline MPFS-1 & 16.46 & 17.20 & 19.03 & 5.15 & 5.22 & 6.15 \\
\hline MPFS-2 & 14.43 & 13.86 & 12.32 & 4.69 & 4.63 & 3.86 \\
\hline MPFS-3 & 9.85 & 9.73 & 9.98 & 2.88 & 2.69 & 3.09 \\
\hline MPFS-4 & 27.17 & 29.76 & 24.16 & 9.65 & 10.52 & 7.51 \\
\hline Cognitive empathy & 44.62 & 44.81 & 44.40 & 4.68 & 4.51 & 4.88 \\
\hline Affective empathy & 37.30 & 37.35 & 37.24 & 4.04 & 3.84 & 4.27 \\
\hline Total empathy & 81.92 & 82.16 & 81.64 & 8.39 & 8.01 & 8.84 \\
\hline $\mathrm{N}$ & 322 & 173 & 149 & 322 & 173 & 149 \\
\hline
\end{tabular}

$\mathrm{S}=$ Entire sample, $\mathrm{Y}=$ Younger sample, $\mathrm{O}=$ Older sample

\section{Statistical Analyses}

The descriptive statistics in Table 2 highlighted several mean differences between younger and older participants. As this study was largely exploratory, cases where results failed to reach critical value significance, but came close enough to be considered "just barely" or "almost" significant, were treated as "marginally significant" in our interpretation. It has been suggested that marginally significant $(p<.2)$ results in exploratory studies can be relevant, and, therefore, merit discussion (Vogt, 2000).

Although younger participants showed greater means on every level of empathy, significant age differences in empathy were not found: cognitive empathy $t(320)=.790, p=.430, d=0.09$; affective empathy $t(320)=$ $.246, p=.806, d=0.03$; total empathy $t(320)=.558, p=.557, d=0.06$. As equal variance was not assumed for the younger/older classification, Welch's $t$ was used for the comparison. Significant differences between younger and older participants were found for music preferences, with younger participants significantly preferring MPF-2, $\mathrm{t}(320)=3.24, p=.001, d=0.33$, and MPF-4, $t(320)=5.55, p$ $<.001, d=0.61$. Older participants significantly preferred MPF-1, $t(320)=-2.85, p=.005, d=0.32$, and older participants preferred MPF-3, but the age difference was not significant, $t(320)=-.736, p=.446, d=$ 0.09 .

\section{Relationship Between MPFS and BES Scores}

Bivariate Pearson's correlation coefficients were calculated for each sample (i.e. entire, younger, and older), on all pairs of variables of interest. For the entire sample, MPF-1 $(r=.07)$ and MPF-2 $(r=.09)$ had stronger associations with empathy than MPF-3 $(r=-.06)$ and MPF-4 $(r=.03)$. This was the same pattern when splitting the sample by age. For the younger sample, MPF-1 $(r=.03)$ and MPF-2 $(r=.07)$ had stronger associations with empathy than MPF-3 $(r=-.04)$ and MPF-4 $(r=.03)$. For the older sample, MPF$1(r=.05)$ and MPF-2 $(r=.11)$ had stronger associations with empathy than MPF-3 $(r=.01)$ and MPF-4 $(r$ $=.09)$.

\section{Regressions}

In addition to the "relationship-descriptive" qualities, the above correlation coefficients were also obtained for the purpose of prediction. Regression models were used to predict empathy values from scores on the MPFS. For the entire sample, marginally significant regression models were produced on MPF-2 $\left(r^{2}=.007\right.$, $\beta=.085, F[1,320]=2.35, p=.127)$, and MPF-4 $\left(r^{2}=.007, \beta=.083, F[1,320]=2.20, \mathrm{p}=.139\right)$ with all facets of empathy. When splitting the sample by age, the expected results were obtained; specifically in younger males. Cognitive empathy $\left(r^{2}=.084, \beta=.290, F[1,45]=4.12, p=.048\right)$, affective empathy $\left(r^{2}=\right.$ 
$.161, \beta=.402, F[1,45]=8.66, p=.005)$, and total empathy $\left(r^{2}=.162, \beta=.403, F[1,45]=8.72, p=.005\right)$. For the younger females, only marginally significant models were produced, and only for MPF-2 on cognitive empathy $\left(r^{2}=.015, \beta=.122, F[1,126]=1.87, p=.174\right)$. For the older participants, the regression models reflected the entire sample's models (i.e. marginally significant on MPF-2: $r^{2}=.006, \beta=.114$, $F[1,148]=1.95, p=.165 \&$ MPF-4: $\left.r^{2}=.004, \beta=.102, \mathrm{~F}[1,148]=1.54, \mathrm{p}=.217\right)$.

\section{Hypotheses}

The hypothesized age differences in empathy were not supported. Younger participants scored higher on every level of empathy (cognitive empathy $M_{\text {younger }}=44.81$, affective empathy $M_{\text {younger }}=37.35$, total empathy $M_{\text {younger }}=82.16 ; c f$. cognitive empathy $M_{\text {older }}=44.40$, affective empathy $M_{\text {older }}=37.24$, total empathy $\left.M_{\text {older }}=81.64\right)$. However, the mean differences were non-significant $(t[320]=.790, p=.430$; $t[320]=.246, p=.806 ; t[320]=.558, p=.557$ respectively). Only on factors of preferred music was there a significant age difference. Whereas older participants preferred music associated with MPF-1 $\left(M_{\text {older }}=\right.$ $\left.19.03 c f . M_{\text {younger }}=17.20, t[320]=-2.85, p=.005\right)$, younger participants preferred music associated with MPF-2 $\left(M_{\text {younger }}=13.86, c f . M_{\text {older }}=12.32, t[320]=-3.24, p=.001\right)$, and MPF-4 $\left(M_{\text {younger }}=29.76, c f . M_{\text {older }}\right.$ $=24.16, t[320]=5.55, p<.001)$. There was no significant age difference on MPF-3 $\left(M_{\text {younger }}=9.73, c f\right.$. $\left.M_{\text {older }}=9.98, t[320]=-.763, p=.446\right)$.

Part of the main hypothesis was that the four MPFS factors would have unique associations with empathy. Specifically, it was hypothesized that preferences for MPF-1 and MPF-2 would have stronger associations with cognitive and total empathy than preferences for MPF-3 and MPF-4. Pearson's correlations showed that, after controlling for age and gender, MPF-1 and MPF-2 did show greater associations with empathy ( $r=.07$, and $r=.09$ respectively) than did MPF-3 and MPF-4 $(r=-.06$, and $r=$ .03 respectively). Again, the data reflected these hypotheses, without achieving statistical significance. At this stage, the differences in the music preference factor-empathy association for the entire sample can only be explained in terms of random variability. Finally, it was hypothesized that age would influence the variance in the music preference factor-empathy association. In support of this hypothesis, the models with cognitive- and total empathy as the criteria were significant at $p<.01$ level. Affective empathy, on the other hand, only produced a significant multiple regression model on MPF- - at $p<.05$, all of which had effect sizes $\left(f^{2}\right)$ between .02 and .10 (i.e. small to medium).

\section{DISCUSSION}

The aims of this exploratory study were to examine: (a) differences between late adolescents and adults for music preferences and empathy; (b) in what capacity (i.e. strength and direction) music preferences might be related to empathy; (c) whether age and/or gender are mitigating factors in those relationships; and (d) how well preferred music genre is able to predict cognitive, affective, and overall levels of empathy as an indicator of prosocial behaviors.

\section{Music Preference and Empathy Differences Between Late Adolescents and Adults}

The results showed that there was a significant age difference in music preference between late adolescents and adults. Whereas older participants preferred music associated with MPF-1 (reflexive and complex), younger participants preferred music associated with MPF-2 (intense and rebellious).

Regarding the age difference in empathy, previous research (e.g. Eisenberg \& Fabes, 1998; Nantel-Viver et al., 2009) posits that empathic concern matures during late adolescence. Total empathy scores of the younger participants, the late adolescents, in the current study may reflect this proposition, since the younger group's total empathy mean was higher than that of the full sample mean. This suggests that between the ages of 18 and 24 participants' empathy may be greater than those aged 25 years and older. The finding that younger participants scored higher for this trait than older participants may indicate that the transition from late adolescence into adulthood may be accompanied by a decrease in empathic concern, and thus prosocial behaviors.

So it seems that as children resemble Machiavelli's Prince (Hay, 1994) by learning how not to be good (i.e. decreasing their prosocial behaviors), so too do late adolescents decrease their prosocial behaviors as they transition into adulthood. We argue that, as the responsibilities and stresses of life 
increase with adulthood, an individual's own life concerns may occupy the majority of their available cognitive energy, leaving less for the processing of, and attending to, the emotional concerns of others. It appears that, even though adults are capable of having their empathic concern increased (Greitemeyer et al., 2010), without such intentional intervention, this may not usually happen. This was not a longitudinal study and so it is possible that the results may reflect generational differences, with participants in these two groups having been exposed to different enculturating forces with resultant difference impacts on prosocial behaviors and/or musical taste. However, despite the lack of longitudinal evidence here, the results of the present study may be taken to suggest that as late adolescents transition into adulthood, their empathic concern decreases (and with it, presumably, their highly correlated prosocial behaviors).

\section{The Relationship Between Music Preferences and Empathy}

Music preferences have been found repeatedly to predict behavioral patterns (e.g. Selfhout et al., 2009; Rentfrow \& Gosling, 2006; Leung \& Kier, 2008; Mulder et al., 2010), identity formation (e.g., Wester et al., 1997; Sprankle \& End, 2009), and adolescent psychopathology (e.g., Miranda \& Claes, 2008; Schwartz \& Fouts, 2003; Martin et al., 1993). The results of the current study add credence to previous findings concerning music preferences' predictive utility, in this instance concerning individual-difference characteristics (i.e. empathy), specifically for males, and across developmental stages. When empathy was regressed onto each MPFS factor, it was predominately the younger males that produced significant models, and only for reflexive and complex, and intense and rebellious music. We postulate that a ceiling effect for female empathy rendered the collective group's regression models non-significant, especially considering that females in these samples outnumbered males 3:1. Removing females from the samples revealed the expected effects in males.

Similarly, music preferences accounted for empathy variance in significant regression models. Reflexive and complex (MPF-1), and intense and rebellious (MPF-2) music explained the most empathy variance of all the music factors, across age. It is thought that the lyrical content of genres in these two factors is far broader (i.e. covering a greater number of topics or issues) on an emotive level than that of MPF-3 and MPF-4, especially in terms of the issues that plague adolescents (Leung \& Kier, 2008). Upbeat and conventional music (MPF-3) took variance away from empathy and was negative in its association. The negative association that MPF-3 had with empathy could also be explained in terms of lyrical content. Music genres encompassed by MPF-3 generally lack the same broad emotive content, tending to pertain instead to boy-girl interactions, materialism/consumerism, or self-centered and mundane content (Clark \& Giacomantonio, 2013; Leung \& Kier, 2008).

Furthermore, it was shown that all four music preference factors had stronger associations with cognitive empathy than with affective and/or total empathy. We suggest that understanding the lyrical content of music is more akin to taking the perspective of the musician as another person, as opposed to merely feeling the musician's emotive state vicariously. For example, listening to a song that makes you cry may not necessarily be a reflection of how the composer felt; some may cry simply because of the accidental context of the listener's own life when he or she first heard the song. As such, it is possible that music preferences have a more relevant association with the cognitive aspects of social and moral development than the affective aspects.

The results of the current study also add credence to previous findings concerning sex differences in music preferences. We showed that male participants had stronger preferences for reflexive and complex music, and intense and rebellious music (i.e. MPF-1 and MPF-2, which incorporate classical, jazz, heavy rap, and metal music). In addition, female participants had stronger preferences for music that is upbeat and conventional, and energetic and rhythmic (i.e. MPF-3 and MPF-4, which incorporate pop, urban, dance, and radio music). Recent studies highlighting the sex differences in developmental issues (Schwartz \& Fouts, 2003; Martin et al., 1993) contribute to the following explanation. Females are thought to focus more on appearance, relationships, and issues of acceptance, which are themes embedded in MPF-3 and MPF-4 (Leung \& Kier, 2008), which we suggest may explain the female preferences found in the present study. Conversely, males are thought to focus more on individualization and angst issues, which are themes embedded in MPF-1 and MPF-2 (Leung \& Kier, 2008). This, we suggest, explains male preferences for the aforementioned music factors. 


\section{CONCLUSION}

In conclusion, this study showed that each of the music preference factors highlighted here has unique associations with empathy that differ across younger (18-24 years old) and older ( $\geq 25$ years old) participants. Specifically, it was shown that music genres encompassed by MPF-1 and MPF-2 are stronger in their associations with empathy than are those encompassed by MPF-3 and MPF-4. In fact, MPF-3 was negatively associated with empathy, indicating that those who have greater preferences for these genres may be lower in empathic concern. Additionally, MPF-4 was shown to have very little influence on empathy, positively or negatively, indicating that these genres of music contain little to no emotive messaging influencing empathic concern - a finding reflected in previous research (Clark \& Giacomantonio, 2013; Leung \& Kier, 2008). In addition, age differences concerning empathy and music preferences were present, indicating that empathy decreased with the onset of adulthood, and that music preferences in adulthood no longer reflect the social, moral, and developmental issues that plague late adolescents.

The most interesting finding, however, concerns the systematic positive associations that music preference has with the cognitive component of empathy compared with the affective and overall components. This finding invites us to consider whether music preferences are more relevantly associated with cognitive aspects of development than affective ones. In addition, these findings suggest that future research into the developmental relevance of music preferences might focus more closely on cognitive rather than affective constructs.

Furthermore, the importance of the demonstrated influence of music preference on empathy should not be underestimated, especially given the aforementioned established correlation between empathy and prosocial behavior. It is postulated that individuals with music preferences that have greater associations with empathy (i.e. reflexive and complex, and intense and rebellious) may be more likely to exhibit greater prosocial behaviors than those with preferences for music that has negative or no association with empathy (i.e. upbeat and conventional, and energetic and rhythmic). Further research would be needed to validate the current findings. However, if this is the case, the way in which popular commercial music is censored, along with ratings of music television shows (i.e. according to explicit language and sexual content only), may have to be reviewed to incorporate socio-emotional influences given the association between music and empathy.

Finally, in considering this study in terms of empathic concern being positively related to social intelligence, social competence, and other aspects of psychosocial adaptation during adolescence, it is thought that music genres pertaining to greater developmental, sociological, ideological, and political messaging (such as MPF-1 and MPF-2; essentially those linked in this study with greater empathy association)-opposed to narrow-focused messaging - may promote, through exposure, a greater understanding of a diversity of concepts in the listener (Clark \& Giacomantonio, 2013). Exposure to such music genres may serve as a buffer for all forms of aggressive and antisocial tendencies in adolescence (Albiero, et al., 2009; Miranda \& Claes, 2008), and foster the development of strategies and support mechanisms (cognitive) that help to make positive, rational choices (Packer \& Ballantyne, 2011). Furthermore, this exposure may allow the listener to experience greater self-exploration, validation, and normalization of issues, thus enhancing his or her social development, essentially leading to a more productive and fulfilling life.

\section{Limitations of The Current Research}

There are several limitations to the present study. The demographics of the sample were not ideal. Females were largely over-represented. This, we suggest, may have led to inflated means for the empathy scores of the sample as a whole. We postulate that this female over-representation led to the lack of outright significance we found for the majority of simple regression models. Furthermore, due to time and ethics restrictions we were unable to include early adolescents in the study (12-17 years old). This adds an extra element of caution to the interpretation and generalization of our results and their implications. However, given the exploratory nature of the research, this caution is welcomed. There were issues surrounding the interpretation of the developmental significance of music preferences as measured. Since this sample contained age groups beyond early adolescence, a retrospective assessment of participants' music preferences may have highlighted the depth or strength an individual has for that preference. With regard to 
measuring empathy, the BES is quite robust in its psychometric properties (Alberio et al., 2009; Jolliffe \& Farrington, 2006b). However, we wonder whether we might be better served by incorporating measures of peer assessments of an individual's empathic concern (or some other post hoc confirmatory assessments). Finally, this study was only correlational, which does not allow causal conclusions. However, the exploratory nature of this study lent justification for choosing this design, as the focus was to highlight relationships between un-researched variables.

\section{Suggestions for Future Research}

First, we are quite pleased with the MPFS' statistical ability to capture music preferences equally to that of existing measures (i.e. the STOMP), especially considering that the MPFS pertains to greater logical content and face validity, in that it captures music preference as a measure of time spent intentionally listening to a specific genre, as opposed to a mere rating of a factor-analyzed banner genre. Further psychometric validation of the MPFS (which is currently underway) would highlight its greater relevance for music preference assessment. In addition, studies using the MPFS as an assessment tool would add validity to their results.

Future research into empathy and music preference associations should also improve upon the limitations of the current study, namely to incorporate peer assessments into measures of empathy, and the retrospective assessment of music preferences for participants beyond early adolescence. Extending empathy measures in this way may result in a more robust assessment of the construct for future research, especially when samples are gender- and/or age-biased, and a retrospective assessment of music preferences for participants who have transcended the identity formation period may give researchers greater knowledge of the developmental relevance of the favored youth culture pastime, intentional music listening. In addition, future research should include early adolescents to ensure the entire developmental period is investigated.

The question of impact on empathy via music exposure remains an important one. Future research into the developmental relevance of music listening may consider an experimental study similar to Greitemeyer et al., (2010), in which it was suggested that exposure to prosocial media increased empathy. Future research exposing participants to music factors, which have been suggested in this study to have greater associations with empathy (i.e. MPF-2, intense and rebellious), may examine more closely the music preference-empathy relationship.

Furthermore, as this study has highlighted, it appears that music preferences may be more relevant to cognitive as opposed to affective components of development. This is thought to be a function of the metaphoric, figurative, and poetic nature of music lyrics, and the cognitive processes needed to decipher meaning. Further research into music preferences may investigate possible links with constructs such as verbal comprehension, academic results, overall intelligence (i.e. IQ), or possibly any construct relating to the development of an individual's executive functioning, since listening to specific genres of music may promote cognitive processes that lead to greater intelligence (Clark \& Giacomantonio, 2013).

\section{NOTES}

[1] Correspondence can be addressed to: Mr. Shannon S. Clark, Research Scholar, Australian Catholic University, c/- School of Psychology McAuley Campus, 1100 Nudgee Road, Banyo, QLD 4014, Australia. E-mail: olskuw@gmail.com

\section{REFERENCES}

Albiero, P., Matricardi, G., Speltri, D., \& Toso, D. (2009). The assessment of empathy in adolescence: A contribution to the Italian validation of the 'Basic Empathy Scale'. Journal of Adolescence, 32(2), 393-408.

Australian Associated Press. (2011, January 15). Volunteers turn out for recovery in Brisbane as flood crisis spreads across country. The Australian. Retrieved March 8, 2011, from $\mathrm{http}$ ://www.theaustralian.com.au/in-depth/queensland-floods/volunteers-turn-out-for-recovery-in-brisbaneas-flood-crisis-spreads-across-country/story-fn7iwx3v-1225988266655 
Australian Bureau of Statistics. (2008). Volunteer Work Survey [Fact sheet]. Retrieved March 29, 2011, from http://www.ausstats.abs.gov.au/ausstats/subscriber.nsf/0/C5286282C082577CA2373100019861/ \$File/44410_2006.pdf

Caravita, S., Di Blasio, P., \& Salmivalli, C. (2009). Unique and interactive effects of empathy and social status on involvement in bullying. Social Development, 18(1), 140-163.

Clark, K. (1980). Empathy: A neglected topic in psychological research. American Psychologist, 35(2), 187-190.

Clark, S., \& Giacomantonio, S. G. (2013). Music preferences and empathy: Toward predicting prosocial behavior. Psychomusicology; Music, Mind, and Brain, 23(3), 177-186.

Cohen, D., \& Strayer, J. (1996). Empathy in conduct-disordered and comparison youth. Developmental Psychology, 32, 988-998.

Colley, A. (2008). Young people's musical taste: Relationship with gender and gender-related traits. Journal of Applied Social Psychology, 38(8), 2039-2055. doi: 10.1111/j.1559-1816.2008.00379.x

Crenshaw, D., \& Hardy, K. (2007). The crucial role of empathy in breaking the silence of traumatized children in play therapy. International Journal of Play Therapy, 16(2), 160-175.

Davis, M. (1980). A multidimensional approach to individual differences in empathy. JSAS Catalog of Selected Documents in Psychology, 10, 85.

Davis, M. (1983). Measuring individual differences in empathy: Evidence for a multidimensional approach. Journal of Personality and Social Psychology, 44(1), 113-126.

de Kemp, R., Overbeek, G., de Wied, M., Engels, R., \& Scholte, R. (2007). Early adolescent empathy, parental support, and antisocial behavior. The Journal of Genetic Psychology: Research and Theory on Human Development, 168(1), 5-18.

Dunn, P., de Ruyter, B., \& Bouwhuis, D. (2012). Toward a better understanding of the relation between music preference, listening behavior, and personality. Psychology of Music. 40, 411-428.

Eisenberg, N., \& Fabes, R. A. (1998). Prosocial development. In W. Damon, \& N. Eisenberg. Handbook of child psychology: Vol. 3. Social, Emotional and Personality Development (5th ed., pp. 701-778). New York: Wiley.

Epley, N., Savitsky, K., \& Gilovich, T. (2002). Empathy neglect: Reconciling the spotlight effect and the correspondence bias. Journal of Personality and Social Psychology, 83(2), 300-312.

Feeney, K. (2011, January 21) List of flood relief events to be held across Brisbane. The Brisbane Times. Retrieved March 29, 2011, from http://www.brisbanetimes.com.au/entertainment/list-of-flood-reliefevents-to-be-held-across-brisbane-20110121-19z1a.html

Ferrier, T. (2011, January 18). 'No truth' to volunteer's claim he found women's bodies after Queensland floods. The Herald Sun. Retrieved March 29, 2011, from http:/www.heraldsun.com.au/news/specialreports/bodies-found-in-queensland-clean-up/storyfn7kabp 3-1225990220854

George, D., Stickle, K., Rachid, F., \& Wopnford, A. (2007). The association between types of music enjoyed and cognitive, behavioral, and personality factors of those who listen. Psychomusicology, 19, 3256.

Geter, T. \& Streisand, B. (1995). Recording sound scales: The music industry rocks and rolls to the newest financial rhythms, U.S. News and World Report, $25^{\text {th }}$ September, pp 67-68, 70, 72. 
Godfrey, M., Hutton, C., \& Marszalek, J. (2011, January 15). Army of volunteers roll up sleeves in Qld. The Brisbane Times. Retrieved March 8, 2011, from http://news.brisbanetimes.com.au/breaking-newsnational/army-of-volunteers-roll-up-sleeves-in-qld-20110115-19rly.html

Greitemeyer, T. (2009a). Effects of songs with prosocial lyrics on prosocial thoughts, affect, and behavior. Journal of Experimental Social Psychology, 45(1), 186-190.

Greitemeyer, T. (2009b). Effects of songs with prosocial lyrics on prosocial behavior: Further evidence and a mediating mechanism. Personality and Social Psychology Bulletin, 35(11), 1500-1511.

Greitemeyer, T., Osswald, S., \& Brauer, M. (2010). Playing prosocial video games increases empathy and decreases Schadenfreude. Emotion, 10(6), 796-802.

Hay, F. (1994). Prosocial development. Journal of Child Psychology and Psychiatry, 35(1), 29-71.

Hoffman, L. (2001). Toward a comprehensive empathy-based theory of prosocial moral development. In A. C. Bohart \& D. J. Stipek (Eds.), Constructive and destructive behavior: Implications for family, school, \& society (pp. 61-86). Washington, DC: American Psychological Association.

Huron, D. (2001). Is music an evolutionary adaptation? The biological foundations of music (pp. 43-61). New York, NY: New York Academy of Sciences; US.

Jolliffe, D., \& Farrington, P. (2004). Empathy and offending: A systematic review and meta-analysis. Aggression and Violent Behavior, 9(5), 441-476.

Jolliffe, D., \& Farrington, P. (2006a). Examining the relationship between low empathy and bullying. Aggressive Behavior, 32(6), 540-550.

Jolliffe, D., \& Farrington, P. (2006b). Development and validation of the Basic Empathy Scale. Journal of Adolescence, 29(4), 589-611.

Juslin, N., \& Laukka, P. (2003). Communication of emotions in vocal expression and music performance: Different channels, same code? Psychological Bulletin, 129(5), 770-814.

Juslin, N., \& Sloboda, J. (2001). Music and emotion: Theory and research (pp. viii, 487). New York, NY: Oxford University Press; US.

Kaplan, R., \& Saccuzzo, D (2005). Psychological testing: Principles, applications, and issues (6 ${ }^{\text {th }}$ ed.). Thompson Wadsworth: Belmont, CA.

Kassin, S., Markus, H., \& Fein, S. (2008). Social psychology (8th ed.). Boston, MA: Belmont, CA: Wadsworth/Cengage Learning.

LeSure-Lester, G. (2000). Relation between empathy and aggression and behavior compliance among abused group home youth. Child Psychiatry and Human Development, 31(2), 153-161.

Levitin, D. (2006). This is your brain on music: The science of a human obsession. New York, NY: Dutton/Penguin Books; US.

Leung, A., \& Kier, C. (2008). Music preferences and civic activism of young people. Journal of Youth Studies, 11 (4), p. 445-460.

Machiavelli, N. (1535/2008). The Prince, (Eds. and Trans. Q. Skinner \& R. Price). Cambridge: Cambridge University Press. 
Martin, G., Clarke, M., \& Pearce, C. (1993). Adolescent suicide: Music preference as an indicator of vulnerability. Journal of the American Academy of Child \& Adolescent Psychiatry, 32(3), 530-535.

McCormick, N. (2009, April 9). Is there too much music? The Telegraph. Retrieved March 30, 2011, from http://telegraph.co.uk

Miller, P., \& Eisenberg, N. (1988). The relation of empathy to aggressive and externalizing/antisocial behavior. Psychological Bulletin, 103(3), 324-344. doi: 10.1037/0033-2909.103.3.324

Miranda, D., \& Claes, M. (2008). Personality traits, music preferences and depression in adolescence. International Journal of Adolescence and Youth, 14(3), 277-298.

Mulder, J., ter Bogt, T., Raaijmakers, A., Gabhainn, N., Monshouwer, K., \& Vollebergh, A. (2010). Is it the music? Peer substance use as a mediator of the link between music preferences and adolescent substance use. Journal of Adolescence, 33(3), 387-394.

Nantel-Vivier, A., Kokko, K., Caprara, V., Pastorelli, C., Gerbino, G., Paciello, M., Tremblay, R. (2009). Prosocial development from childhood to adolescence: A multi-informant perspective with Canadian and Italian longitudinal studies. Journal of Child Psychology and Psychiatry, 50(5), 590-598.

Packer, J., \& Ballantyne, J. (2011). The impact of music festival attendance on young people's psychological and social well-being. Psychology of Music, 39(2), 164- 181.

Rentfrow, P., Goldberg, L., \& Levitin, D. (2011). The structure of musical preferences: A five-factor model. Journal of Personality and Social Psychology. pp None Specified

Rentfrow, P., \& Gosling, S. (2003). The do re mi's of everyday life: The structure and personality correlates of music preferences. Journal of Personality and Social Psychology, 84(6), 1236-1256.

Rentfrow, P. \& Gosling, S. (2006). Message in a ballad: The role of music preferences in interpersonal perception. Psychological Science, 17(3), 236-242.

Rentfrow, P., McDonald, J., \& Oldmeadow, J. (2009). You are what you listen to: Young people's stereotypes about music fans. Group Processes and Intergroup Relations, 12(3), 329-344.

Roberts, D., Henriksen, L., \& Foehr, U. (2004). Adolescents and media: Handbook of adolescent psychology (2nd ed., pp. 487-521). Hoboken, NJ: John Wiley \& Sons Inc; US.

Schäfer, T., \& Sedlmeier, P. (2009). From the functions of music to music preference. Psychology of Music, 37, $279-300$.

Schwartz, K., \& Fouts, G. (2003). Music preferences, personality style, and developmental issues of adolescents. Journal of Youth and Adolescence, 32(3), 205-213.

Scourfield, J., John, B., Martin, N., \& McGuffin, P. (2004). The development of prosocial behavior in children and adolescents: A twin study. Journal of Child Psychology and Psychiatry, 45(5), 927-935.

Selfhout, M., Branje, S., ter Bogt, T., \& Meeus, W. (2009). The role of music preferences in early adolescents' friendship formation and stability. Journal of Adolescence, 32(1), 95-107.

Sigelman, C., \& Rider, E. (2009). Life-Span human development (6th ed.). Belmont, CA: Wadsworth/Cengage Learning.

Sprankle, E., \& End, C. (2009). The effects of censored and uncensored sexually explicit music on sexual attitudes and perceptions of sexual activity. Journal of Media Psychology: Theories, Methods, and Applications, 21(2), 60-68. 
Tabachnick, B., \& Fidell, L, (2007). Using multivariate statistics ( ${ }^{\text {th }}$ ed.). USA: Pearsons Education.

Vogt, W. P. (2000). Dictionary of statistics and methodologies: A non-technical guide for the social sciences $\left(2^{\text {nd }}\right.$ ed.). Thousand Oaks, CA: Sage Publications, Inc.

Volunteering Queensland Organisation. (2011) Volunteering trends [Fact sheet]. Retrieved March 29, 2011, from http://www.volunteeringqld.org.au/home/index.php/government/volunteering-trends

Wester, S., Crown, C., Quatman, G., \& Heesacker, M. (1997). The influence of sexually violent rap music on attitudes of men with little prior exposure. Psychology of Women Quarterly, 21(4), 497-508.

Wispé, L. (1972) Positive forms of social behavior: An overview. Journal of Social Issues, 28, 1-20. 